\title{
Reverse Engineering Goal Models from Legacy Code
}

\author{
Yijun $\mathrm{Yu}^{1}$, Yiqiao Wang ${ }^{1}$, John Mylopoulos ${ }^{1}$, Sotirios Liaskos ${ }^{1}$, Alexei Lapouchnian ${ }^{1}$, \\ Julio Cesar Sampaio do Prado Leite ${ }^{2}$ \\ ${ }^{1}$ Dept. of Computer Science, Univ. of Toronto, \{yijun,yw,jm,liaskos,alexei $\}$ @ cs.toronto.edu \\ ${ }^{2}$ Dept. of Computer Science, PUC-Rio, julio@inf.puc-rio.br
}

\begin{abstract}
A reverse engineering process aims at reconstructing high-level abstractions from source code. This paper presents a novel reverse engineering methodology for recovering requirements goal models from both structured and unstructured legacy code. The methodology consists of the following major steps: 1) Refactor source code by extracting methods based on comments; 2) Convert the refactored code into an abstract structured program through statechart refactoring and hammock graph construction; 3) Extract a goal model from the structured program abstract syntax tree; 4) Identify non-functional requirements and derive softgoals based on the traceability between the code and the goal model. To illustrate this requirements recovery process, we refactor requirements goal models from two legacy software code bases: an unstructured Web-based email in PHP (SquirrelMail) and a structured email client system in Java (Columba).
\end{abstract}

\section{Introduction}

A software reengineering process follows a horseshoe model by first recovering lost abstractions (e.g., elements of design and/or requirements) through reverse engineering [2, 21] (also known as design recovery [19]), and then pushing these abstractions forward into low-level implementations through forward engineering. In the initial horseshoe proposal [18] and all subsequent research, the lowest level abstraction is legacy source code, while the highest level abstractions are elements of a software architecture. The reverse engineering process amounts to architecture recovery, while the forward engineering process amounts to architecture-based development.

For more than a decade, the requirements engineering community has proposed and studied goal models [22, 7, 3, 32] as high level abstractions for modeling early requirements. Goals capture stakeholder intentions. By modeling and analyzing them, we can derive functional and

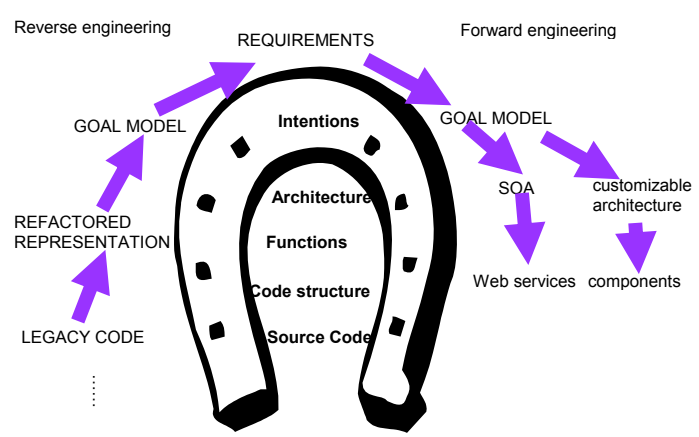

Figure 1. The horseshoe model

non-functional requirements in a systematic and coherent fashion. The goal models developed in this early phase of software development tell us not only the origins of functional and non-functional requirements, but also the space of alternative solutions (operationalizations) that the requirements engineer needs to select from. The KAOS methodology defines the state-of-the-art on this thread of research [7, 32, 33]. Hui et al [17] propose an extended framework for developing requirements that includes modeling and analysis of user goals, skills, and preferences (GSP). The framework is intended for the design of generic, customizable (hereafter high variability) software, to be used by a community of users. The original case for this work involved users with traumatic brain injuries in Oregon State [11].

We are interested in using the GSP framework to reengineer legacy software into generic, high variability software. To meet this objective, we are developing techniques for reverse engineering goal models from legacy software that offers some service (e.g., email). These models can then be revised, refined and extended, so that they can serve as basis for generating an extended version of the legacy software system that supports the same service in multiple ways. This paper presents the reverse engineering phase during which a goal model is extracted from legacy code.

In the sequel, we adapt the horseshoe model as shown in 


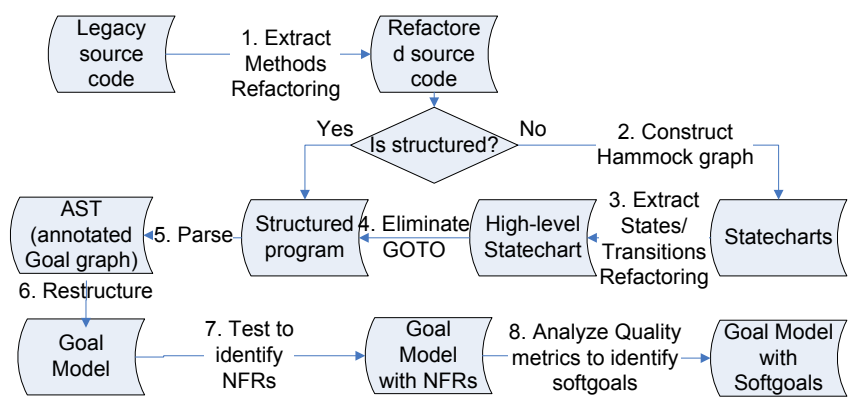

Figure 2. Major steps in our process

Figure 1. Instead of static architecture recovery, we aim at discovering requirements goals from the behaviour of the system, thereby answering the most fundamental of all questions about a software system: What is the system intended for?

Our methodology converts structured and unstructured legacy source code into goal models following the steps illustrated in Figure 2: (1) refactor the source code by extracting methods [12] based on program slicing techniques [35] and programmer comments scattered in the code; if the refactored code is structured then go to step (5); otherwise (2) convert it into equivalent statecharts [14], (3) further refactor the statecharts into higher-level statecharts by extracting states and transitions, and (4) convert the high-level statecharts into an equivalent program which is then structurized by eliminating GOTO's [37]; (5) parse the structured program into an abstract syntax tree (AST) representing an annotated goal graph; (6) restructure the annotated goal graph into requirements goals; (7) identify non-functional requirements (NFR) in the resultant goal model by testing its traceable code in order to (8) derive softgoals from the NFR, using heuristics such as quality metrics.

The rest of the paper is organized as follows. Section 2 presents relevant concepts and the proposed reverse engineering methodology. Section 3 explains the refactoring of source code into its abstract form, and Section 4 explains the extraction of goal models from the refactored programs. Both sections conduct a case study of open-source software systems (SquirrelMail [1] and Columba [9]), showing the independence to programming languages and structureness of the legacy code. Section 5 discusses tool support that reduces the amount of time required for reverse engineering. Section 6 compares our proposal with related work, while section 7 summarizes results and sketches directions for future research.

\section{Concepts and approach}

Our proposal is based on well-founded concepts in software engineering. In particular, goal models $[3,32]$ arise during early requirements elicitation; program slices [35] are useful for program understanding and static analysis; while statecharts constitute a powerful representation for dynamic systems [14]. These concepts are integrated into a methodology through which the gap between goal models and source code is bridged. We first introduce these concepts in more detail, before presenting our proposed methodology.

\subsection{Goal models}

A goal represents a stakeholder intention. A goal can be either fulfilled or not [32], and may depend on subgoals through AND/OR refinements. In [22], softgoals are proposed as means for modeling and analyzing quality attributes. Softgoals, unlike their vanilla cousins, can be partially satisfied or denied, and may depend on other goals and softgoals through MAKES, HELPS, HURTS and BREAKS relations. With goal models, software development proceeds by refining goals, identifying collections of leaf goals that together fulfil root-level goals, and assigning responsibilities for the fulfilment of leaf-level goals.

\subsection{Program slicing}

Unstructured programs can be structured using Hammock graphs $[35,37]$ that have a single entry and a single exit. For structured programs, program slicing [35] generates a slice $P^{\prime}$ of a program $P$ based on a slicing criterion $<p, V>$ where $p$ is a statement of $P$ and $V$ is a subset of the variables of $P$ [31]. Static program slicing finds the statements that are either (control) dependent on $p$ or (data) dependent on $V$. Program dependence graph (PDG) [25] and inter-procedural system dependence graph (SDG) [16] can be seen as results of program slicing, combining both control and data dependence information in a program [10].

\subsection{Statecharts}

Statecharts constitute a concise visual formalism that captures the dynamic behaviour of a system [14]. Statecharts have been adopted in UML as one of the diagrammatic notations for modeling behaviour. Statecharts extend conventional finite state machine diagrams in several ways: a collection of sub-states can be abstracted into a super-state through AND or XOR composition; the number of states is visually reduced by zooming sub-states out; a transition from/to a super-state can abstract a number of transitions from/to its sub-states; parallel AND sub-states also reduce 
the number of combined states. Low-level statecharts carry both control and data dependencies of a program, and are close to implementation; high-level statecharts, on the other hand, hide implementation detail and abstract system behaviour.

Although UML tools such as STATEMATE [15] can generate executable code from given statecharts, it is not yet possible to convert source code into statecharts. Our approach relies on software refactoring and program slicing techniques to do just that.

\subsection{Our approach}

Our approach summarized in Figure 2 is inspired by software refactoring techniques [20]. Refactoring has been proposed [24] as a method for understanding and maintaining complex source code. Refactoring restructures and simplifies source code by improving its internal structure without changing its external behaviour [12]. In our proposal, source code is converted into more abstract form by recursively applying the refactoring operation Extract Method [12]. The scope of this process can be determined by rules of thumb, such as delimiting comments, as these comments often indicate a semantic gap for the program understanding [12]. Aiming at even higher levels of abstraction, the resultant code are subjected to another round of refactoring with a scope determined by Hammock graphs: we extend the Extract Method on an equivalent statechart representation of the program. As with Extract Method, Extract States replaces a sequence of states with a new superstate and Extract Transitions replaces a sequence of transitions with a new transition to the final state. After these refactoring steps, the more abstract statecharts can be converted into an equivalent abstract program further structurized by a GOTO elimination algorithm.

Goal models are then automatically constructed based on the resultant program which is structured and abstract. An annotated goal graph is created from the program AST, and an AND/OR goal model is constructedfrom the annoated goal graph. Using the traceability between code and goal model, we identify non-functional requirements through function tests. By observing the effects on quality metrics through enabling/disabling the identified NFRs, we derive quality softgoals and create proper contribution links from the NFRs to them. The derived softgoals help to bridge the gap between the actual implementation (source code) and its early requirements.

\section{Refactoring for the abstraction of code}

The proposed approach is illustrated with two opensource legacy software systems. The first system, Squirrel Mail 1.5.0 [1], is an unstructured Web-based email client

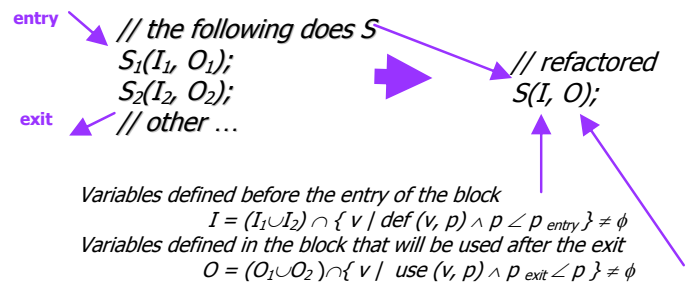

Figure 3. Illustration of Extract Method

implemented in PHP. The second system, Columba 1.0 RC2 [9], is a structured email client implemented in Java. Our approach refactors goal models from both systems, regardless of their different programming languages and environments.

\subsection{Extract Method using comments}

We use Extract Method [12] as a refactoring technique to simplify the legacy code. Extract Method has the advantage that it is applicable to both object-oriented and procedural code. As illustrated in [12], Extract Method deals with statement blocks. Each block is determined by delimitating comments to reveal the programmer intentions. An implicit requirement for Extract Method is that the block must have a single entry and a single exit, and so its structure corresponds to a Hammock graph [37]. Without loss of generality, consider just two statements $S_{1}\left(I_{1}, O_{1}\right)$ and $S_{2}\left(I_{2}, O_{2}\right)$ where $I_{1}, I_{2}$ are the sets of input variables and $\mathrm{O}_{1}, \mathrm{O}_{2}$ are the sets of output variables for the respectively numbered statements $S_{1}$ and $S_{2}$. Note that these statements may also have resulted from a previous application of $E x$ tract Method.

The refactored statement $S(I, O)$ is obtained by program slicing. Specifically, as shown in Figure $3, S$ is the new method named after the comment; $I$ is computed as the set of input variables on entry of the block; $O$ is computed as the set of output variables on exit of the block. We simplify the resultant statement by excluding internal variables of the block that are not used elsewhere in the program (thereby reducing the complexity of the code representation). The Extract Method refactoring step can be applied several times until all the commented blocks are replaced by single statements.

\subsubsection{Refactoring Squirrel Mail}

Squirrel Mail consists of more than 70 KLOC written in PHP. The source code includes the following PHP program files: (1) 37 main PHP programs responsible for generating the Web pages are located in the src subdirectory; (2) 20 PHP routine files called by the load_theme function for customizing different look-and-feel themes. They are lo- 
cated in the themes subdirectory; (3) 15 PHP utility class files located in the classes subdirectory; and (4) 34 PHP utility function files located in the functions subdirectory. In the rest of the paper, we refer to a program file only by its file name; for example, the file src/login.php is referred to as login.php.

Since a dynamic PHP program generates an HTML page with hyperlinks to other dynamic PHP pages, the call to the other PHP program can be delayed until the user clicks at the link. We treat hyperlinks as GOTO statements in an unstructured program. Hence, dynamically generated Webbased programs are considered unstructured, even though PHP is a structured programming language. Therefore, after refactoring Squirrel Mail at step 1 in Figure 2, we need to structurize it through steps 2 to 5 .

For example, the following Hammock graph in login.php has a variable definition SM_PATH exported to the remaining program, while no variable is imported.

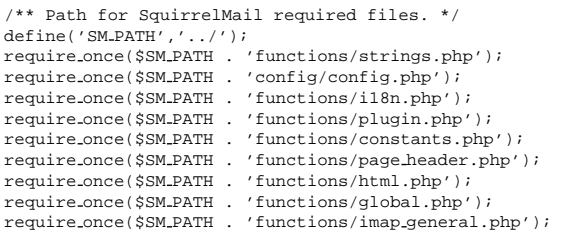

Hence \$SM_PATH constitutes the only output variable of the block, whereas the set of input variables is empty. Note that even global variables that are not used outside the block will be hidden during the abstraction. After slicing analysis, it is safe to declare a new function set_path and call it in login.php as follows.

\$SM_PATH=set_path ();

A complete listing of login.php has 185 LOC. The following listing shows login. php after Extract Method refactoring where all the comments from the original program have been removed. Several blocks have been replaced with method calls.

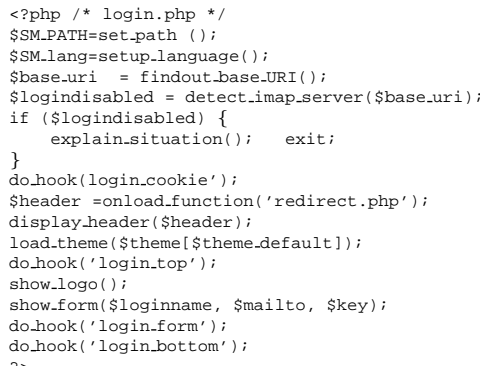

Similarly, such Extract Method refactoring delimited by comments can be applied to the other PHP files.

\subsubsection{Refactoring Columba}

Columba [9] is an open-source Email client that has more than 147 KLOC in Java. The program is structured.
The input to our method is the main class of Columba: org. columba. core.main. Main.run (). The lengthy routine has 81 lines of code. Inside the routine, there are 22 code segments separated by 18 comments and 3 hammock boundaries. The first 20 lines of code are shown below.

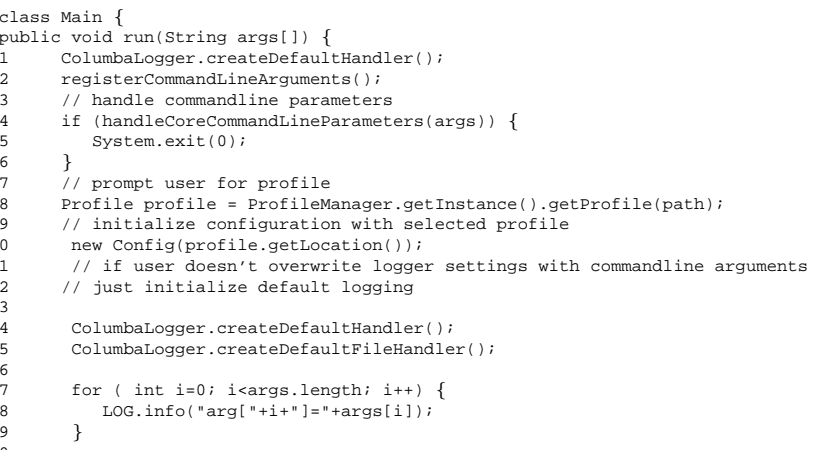

The Eclipse refactoring tool was used to extract 22 methods from the code.

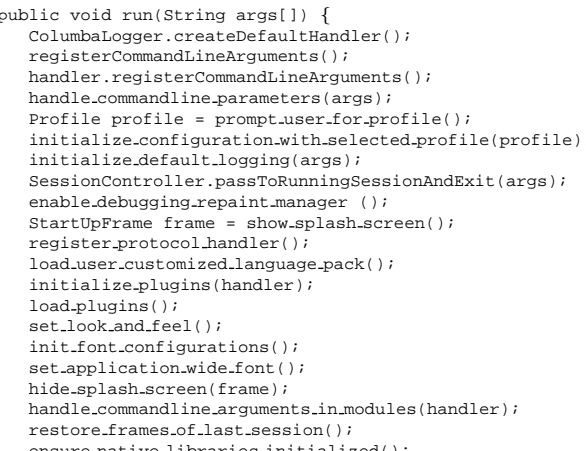

\subsection{Extracting states and transitions using ham- mock graphs}

After the application of the Extract Method refactoring delimited by programmer's comments, the resulting code has no more comments that can be used further. Moreover, due to possible lack of comments accompanied with the program, the refactored code may be still at a lower level of abstraction than the desired requirements goal models.

In Squirrel Mail, the comments are scattered in the routines that the result of the Extract Method refactoring is still at a low level. To make it worse, the unstructureness of a Web-based system, such as Squirrle Mail, limits the extraction of methods to individual routines. Thus, in order to obtain a more abstract representation, a behavioral view of the whole system needs to be extracted. In contrast, the result of Extract Method refactoring on the Columba system is less complex because Columba is structured and it has well-written comments. 


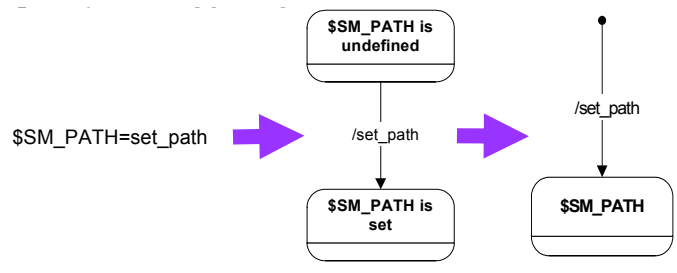

Figure 4. Statechart notations

Static program analysis techniques can help us achieve more abstract program descriptions. In this section, we explain the use of Hammock graphs and statecharts to obtain an abstract view of the system behavior.

Each extracted method has a single entry and a single exit (Hammock graph). At the entry and exit of the Hammock graph, pre- and post-conditions define allowable classes of input/output states. The transition between them is effected by the method. The states and transitions derived from a Hammock graph form a statechart. The statecharts of all hammock graphs are combined into a complete statechart by adding transitions according to the control flow.

For example, in Figure 4, we adopt the statechart notation used in [27]. The action at the transition set_path defines a variable \$SM_PATH. Before the action set_path, the variable \$SM_PATH is undefined. We model undefined variables as initial states. An event can also be put to the left of the slash in the transition label, to specify the triggering condition for the transition. Accordingly, we convert the refactored login.php code into an initial statechart (Figure 5). Note that two special functions do_hook and load_theme can make calls to other methods dynamically. Apart from the static calls, plugin routines registered for a hook name are called dynamically through do_hook. If there are no registered plugins for a hook name, then the action will be a NOP (nil operation). Similarly, the theme routines are called by a load_theme function based on a configuration parameter variable $\$$ theme_default. Note that in this statechart there are two exits, each leading to a different final state.

A statechart constructed from refactored code generally has too many states and transitions, and is hard to understand. We therefore need techniques to group states and transitions into more abstract, and fewer, super-states and super-transitions.

We accomplish this first by introducing layers: a group of states with single entry and exit are grouped together into one super-state. The new super-state replaces the group of the original states comprising it, thereby reducing the number of states. This refactoring step is called Extract States. In Figure 6, we illustrate how three states in the login.php (see Figure 5 and Figure 6a), namely \$SM_PATH, \$SM_lang and \$base_URL, are grouped into

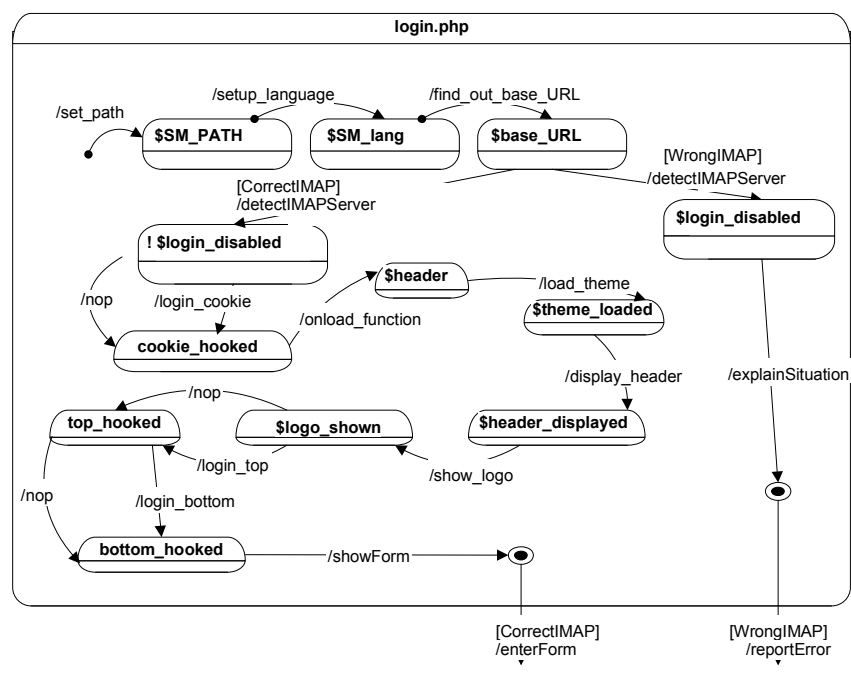

Figure 5. A statechart converted from the refactored code

a super-state named as Globals (Figure 6b). In Figure $7 \mathrm{a}$, the sequence of three transitions in Globals statechart in Figure $6 \mathrm{~b}$ are refactored into a single transition (set_globals).

To simplify the statecharts, a superstate with a single transition inside can be replaced with a new state by merging empty incoming/outgoing transitions to/from the superstate with its internal transition. This refactoring step is called Extract Transition. For example, in Figure 7a, Globals has an empty incoming transition; this transition is merged with set_globals which was inside Globals (Figure 7b). Globals is turned into a new state globalsSet with non-empty outgoing transitions. The result of applying Extract States and Transitions on login.php is shown in Figure 8. Figure 9 shows the result of putting together all top-level programs; the view is then more abstract in Figure 10 to show only the top-level states.

\subsection{Structurizing the statecharts}

The combined statecharts obtained from the PHP programs in the previous steps are unstructured, even though the PHP program has no explicit GOTO statements. On the other hand, a goal model is formed by structured AND/OR decompositions. Before recovering a structured goal model (see Section 4), the statechart needs to be structured to contain only sequences, branches and loop structures.

First, the high-level statechart (Figure 10) can be mapped into a program with GOTO statements. For convenience, in the sequel we use FORTRAN for such programs. Each state 


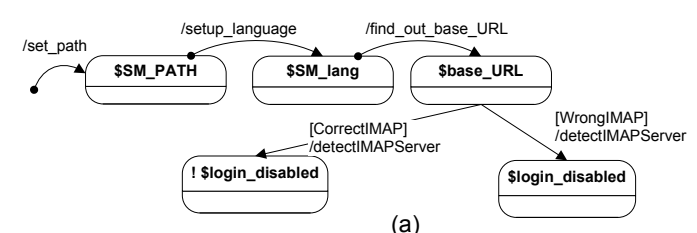

(a)

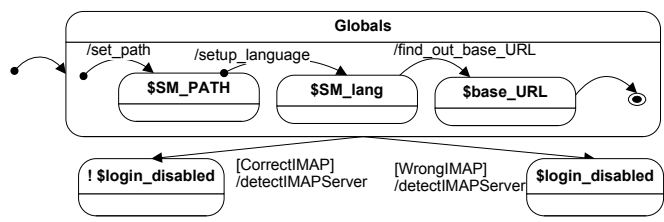

(b)

Figure 6. Extract States Refactoring (a) into (b) where (a) is part of the statechart in Figure 5

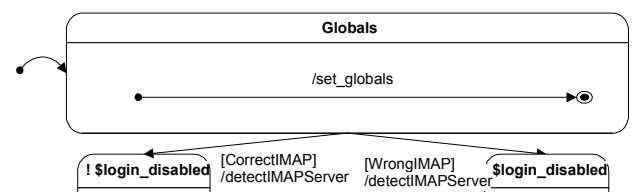

(a)

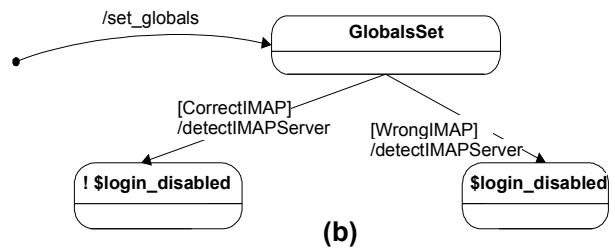

Figure 7. Extract Transitions Refactoring on the new super-state Globals in Figure $6 \mathrm{~b}$

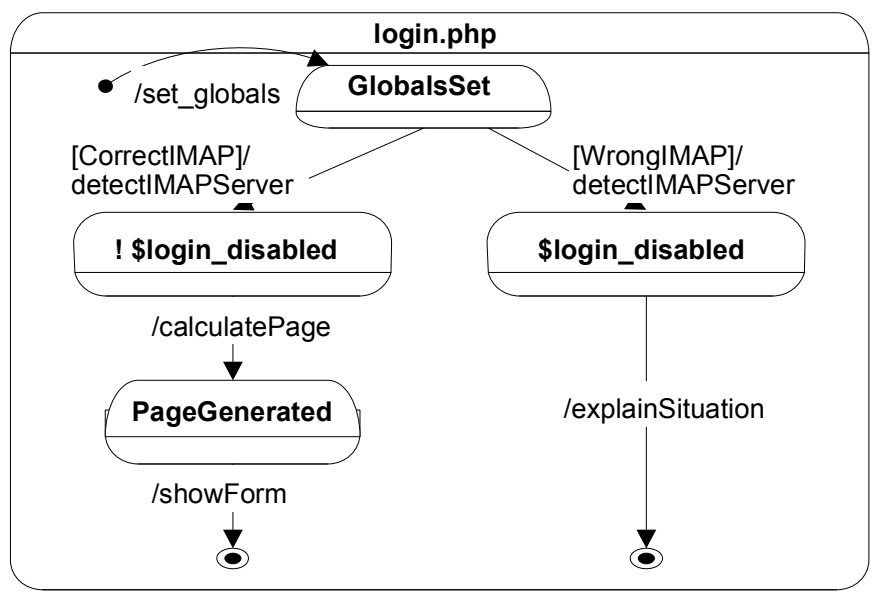

Figure 8. Refactoring on the initial statechart in Figure 7

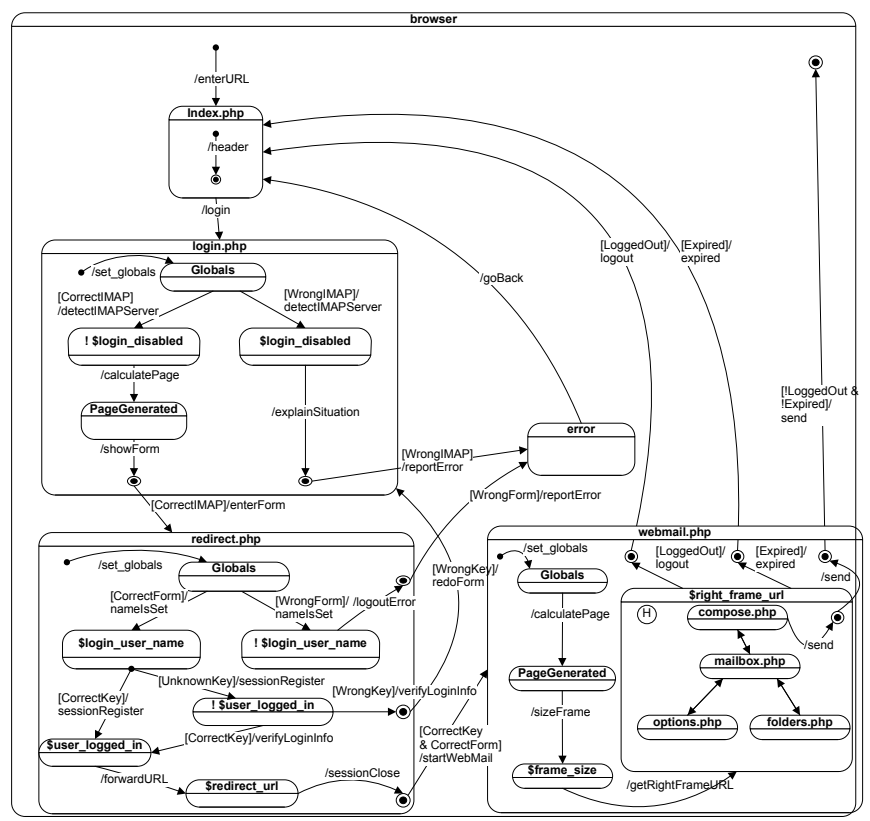

Figure 9. The layered statecharts of the web browser

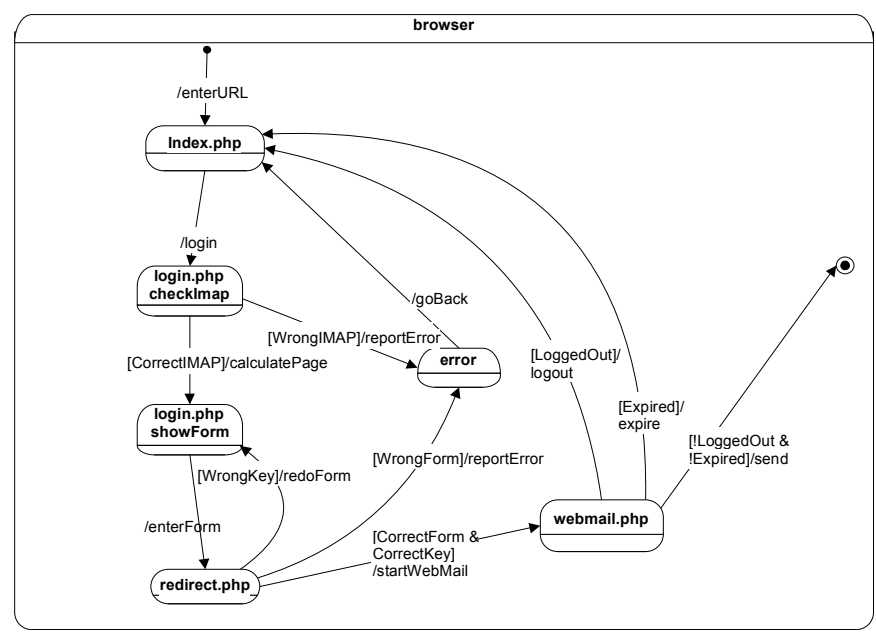

Figure 10. Top-level statechart of the browser 


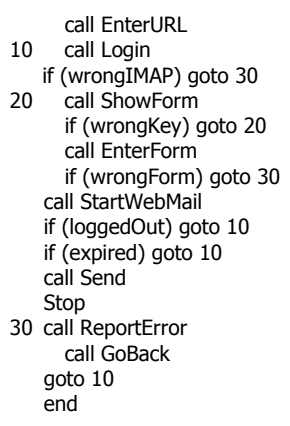

(a)

\section{Figure 11. Structuring the code converted from the statechart in Figure 10}

with more than one entry will be associated with a label, and each transition for an additional exit is associated with a GOTO statement following its activity statement. Secondly, to obtain a structured program, we adopt the GOTO statements elimination implemented in the FPT compiler [37]. It has been established using the theorem prover PVS [26, 28] that all the GOTO's can be removed through semantic preserving transformations, resulting in structured Hammock graphs [37]. As the technique eliminates GOTO's through hammock graph construction, it can be directly combined with our Extract States and Transition refactoring. For example, the statechart in Figure 10 can be converted into a FORTRAN program with GOTO statements (Figure 11a); the program is then structured (Figure 11b) using FPT [8].

\section{Extracting goal models from abstract code}

This section explains the steps 5 to 8 in Figure 2. In the NFR framework [3], a goal has an intended function (intention), and an associated topic (subject matter). In a statechart, the function is found as the action of a state transition and the topic as the contextual state of the statechart. There are two basic modalities for goals: achieve or maintain [32]. In our process, it is easier to identify an achieve goal as a transition between different states whereas a maintain goal as a transition from a state to itself.

As shown in Figure 12, (a) a chain of state transitions designates an AND decomposition of a goal; the transitions correspond to a sequential composite statement. Here an ellipse denotes an unnamed goal. Furthermore, the parallel join of transitions from/to other states (b) designates (a)
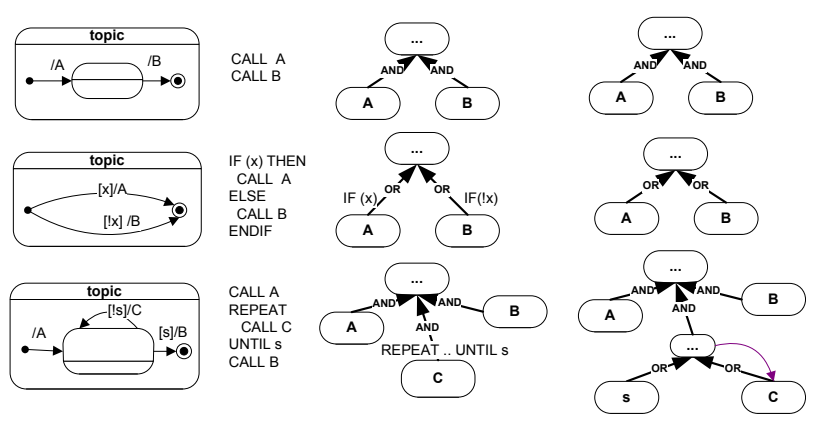

Figure 12. Patterns to extract goal models

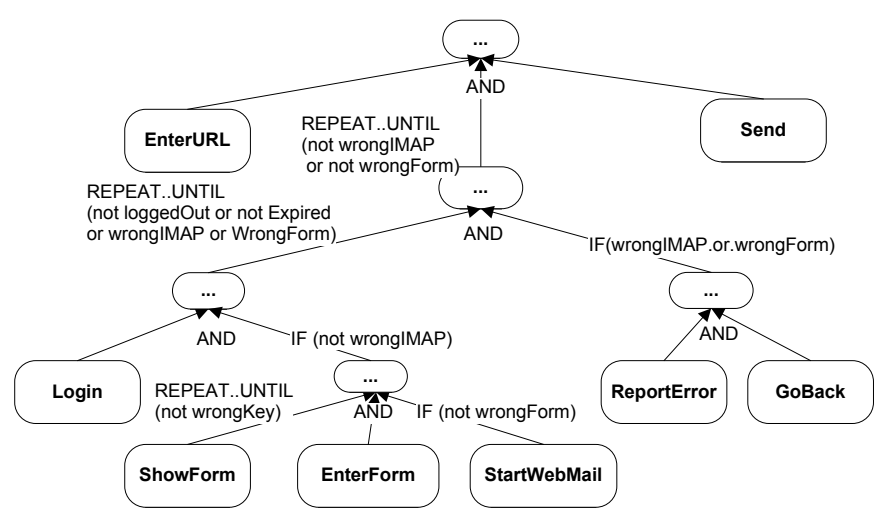

Figure 13. View AST as annotated goal model

OR-decompositions of a goal, corresponding to a branch statement e.g., IF-THEN-ELSE, with a condition derived from the event label on the transitions. Loops can also to be mapped to the goal model where the stop event $\mathrm{s}$ is converted into an intermediate goal along with the actions (c). This case results in a cyclic goal model, where the switching events on the transitions correspond to an OR decomposition. Having a structured state chart/program, we can view its abstract syntax tree (AST) as a goal model annotated with the control conditions, such as $\operatorname{IF}(x)$, $\operatorname{REPEAT}$. .UNTIL (s), etc.

\subsection{Extracting goal models from Squirrel Mail}

The structured program in Figure 11b can be converted into an annotated goal model in Figure 13.

Using the basic conversions (Figure 12) on the annotated control patterns (Figure 13), all the transitions are converted into goals in an AND/OR graph (Figure 14). Moreover, some of the tasks in the goal model are non-functional that contribute to softgoals as qualities or concerns (see the dotted links in Figure 14). For example, "Login" is a non- 


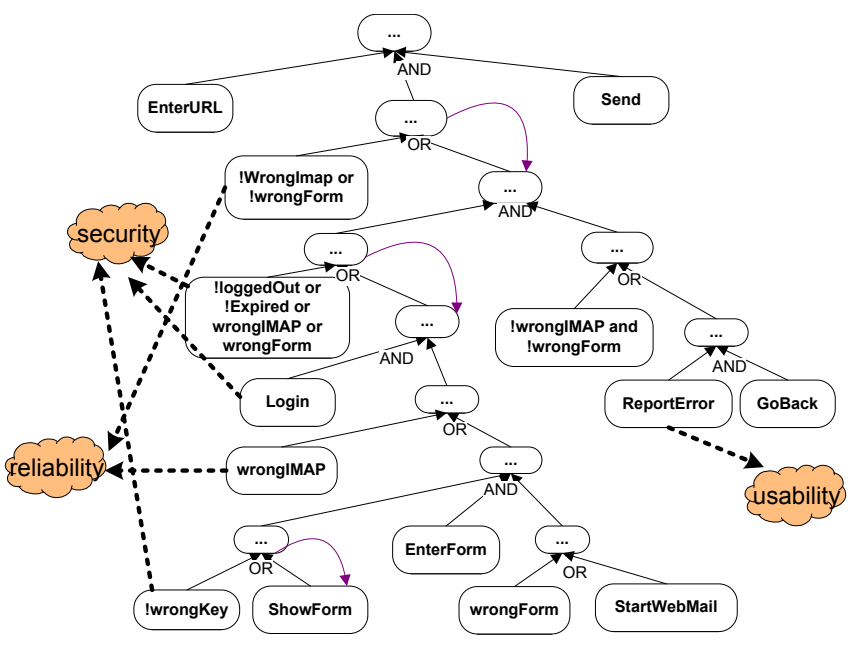

Figure 14. The SquirrelMail goal model

functional task that is concerned by security, "ReportError" is another one that implements the usability concern, etc.

\subsection{Extracting goal models from Columba}

The AST of the further refactored high-level Columba code generates 22 leaf subgoals in the annotated goal model. After applying the three transformation patterns (Figure 12) on the annotated goal graph, we obtain an AND/OR goal model that contains 22 leaf-goals. Among them, 13 goals were identified as non-functional through a functional testing when they are disabled. These NFR goals can be enabled by satisfying guard conditions in the IF statements, which are based on the quality metrics of 9 softgoals, including usability, maintainability, extensibility, etc. One can further catagorize them into decomposing hierarchies.

Having the refactored code in Section 3.1.2, we obtained 22 goals through the AST conversion. But, these goals are not all necessary to run the program. For example, after one can comment out as many as 13 lines of the program, without breaking the system.

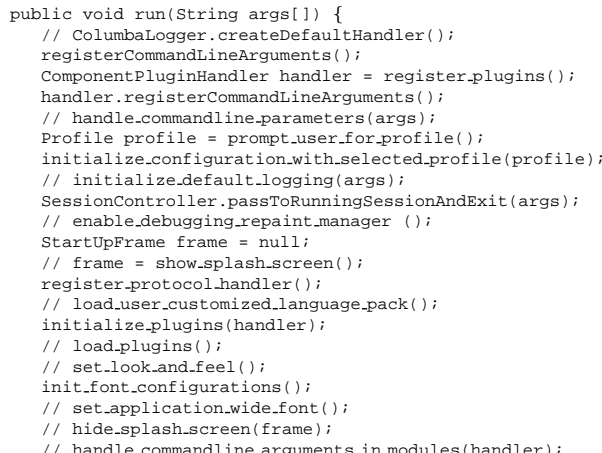

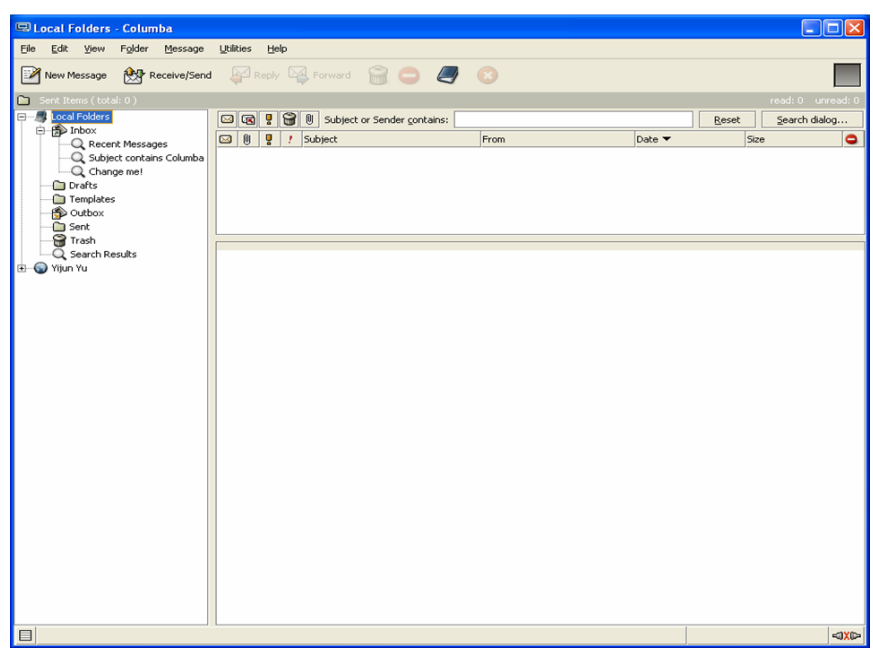

Figure 15. The full-fledged Columba system

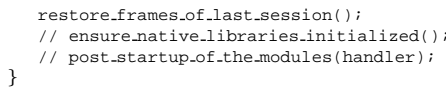

Of course, one may recognize that the program has less usability (as the look and feels and fonts are not as nice as before), becomes more difficult to maintain (as the logging and debugging information are not stored), and harder to extend ( as the plugins are not loaded and the native libraries are not initialized). Figure 16 shows a screenshot of resulting program. Comparing to the full-fledged system in Figure 17, one can see that the look-and-feel is basic and logging is no longer stored after our change. However, the system is still functional: one can still fulfill the goal of sending an email by using the abridged program.

In a further study, we also relate the identified NFRs with the softgoals. We show the refactored code corresponding to the extracted goal model in Figure 17, where the nonfunctional goals are separate into decomposition hierarchy for softgoals.

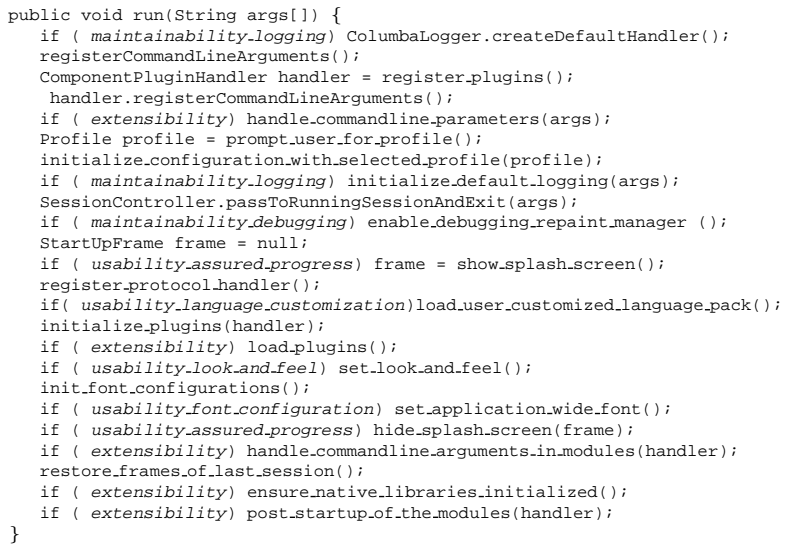




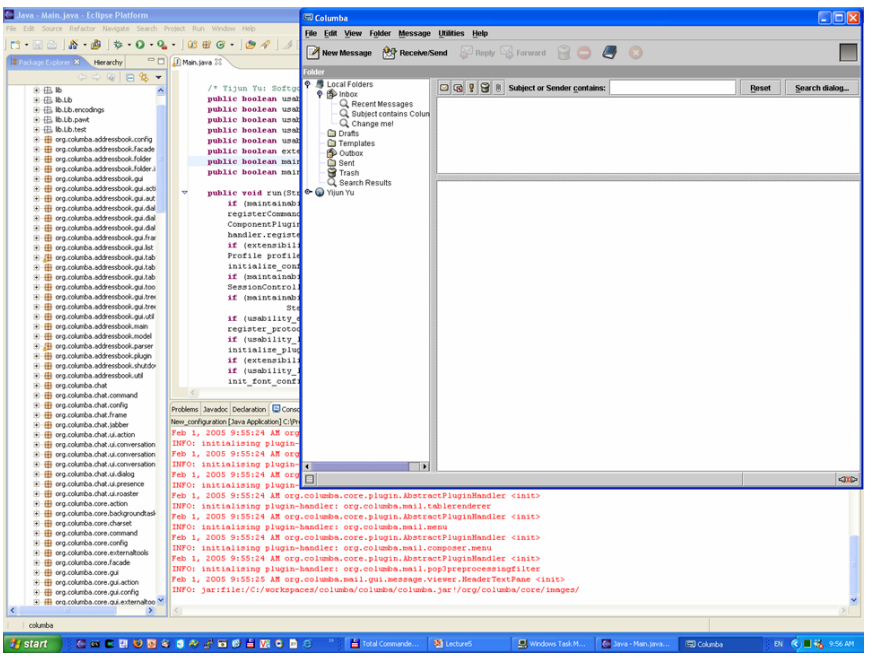

Figure 16. The Columba system with NFR goals disabled

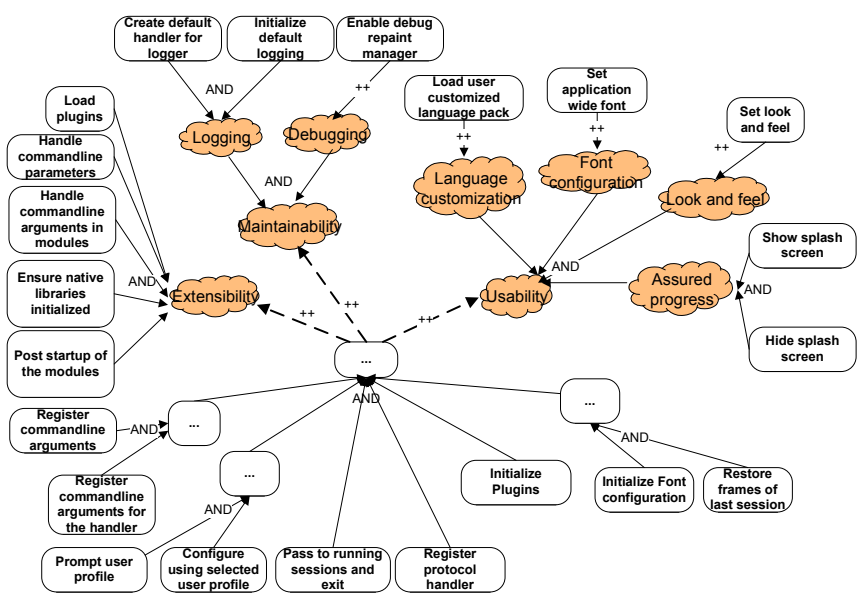

Figure 17. The Columba goal model
These softgoals fill in a gap between the code and its early requirements.

\section{Discussion}

In this section, we outline the implemented tool support for the reverse engineering process and cross-verify our approach through another case study.

First, it is important to check the correctness of the refactoring steps to ensure that semantics is indeed preserved. A standard way for accomplishing this [12] is to test each refactoring step using available test cases. However, test cases can identify incorrectness, but can't ensure correctness. In order to prove that each step is semanticspreserving, we use program slicing techniques to ensure both Extract Method and Extract States and Transitions are properly used. Also, the structuring of the statecharts is based on a well-established theory for GOTO eliminations, where the basic transformations have been proven correct. We can also annotate the state transitions in the statechart with the corresponding code. Therefore the very detailed statechart is exemplified by the program code. Moreover, such traceability allows a change in the high-level abstraction to be reflected in the change of the code.

We can significantly improve the usefulness of our method by automating parts of it as follows:

- Refactoring based on program slicing. We can use the Extract Method as it is available for Java through the Eclipse IDE [36] (Shift_Alt_M). This refactoring is done semi-automatically by selecting statements delimited by programmer comments. Although a general refactoring tool for PHP is not available yet, we are developing an Extract Method tool for PHP and we are looking for a suitable case study in JSP that allows us to reuse existing Java refactoring tool support in Eclipse. Developing a tool for the Extract States and Transitions refactoring is also in our tool implementation agenda.

- Statechart structuring. Currently we deal with the problem by converting statecharts into an equivalent Fortran code with GOTO statements to leverage an existing Fortran compiler that has implemented the GOTO elimination algorithm [8, 37].

- Extracting goal model. The AST of the structured program is used to generate the annotated goal models. The format of these generated goal models conforms to the OMG XMI standard, which is exchangeable with other modeling tools such as EclipseUML or RationalRose. To this end, we used Eclipse modeling framework (EMF). Then we used the JDT API in Eclipse 
to convert any structured Java program into an annotated goal model, and further created an AND-OR goal model using the basic patterns. The annotation labels are automatically transformed into a purely AND-OR goal model using the basic patterns in Figure 12.

- Identifying non-functional goals. Currently, this step is semi-automatic. We obtain the NFR based on the traceability of a goal and a statement in the program such that the statement is guarded by its truth value. Once a goal is considered non-functional, the statement is disabled during execution. We then test the recompiled system to verify that system function is still being delivered. If so, then the goal is tagged as nonfunctional.

- Linking non-functional goals to softgoals. Once nonfunctional tasks are identified, one can resort to the NFR framework [3] to categorize them with certain quality attributes as softgoals in the extended goal model. The quality attributes answer why the nonfunctional tasks are present in the source code.

\section{Related work}

Initially, goal models [7] were proposed to capture requirements, i.e., the optative statements of the environment [33]. Goal models have been extended to represent both functional and non-functional requirements for the software developing processes [22]. The requirements engineering community has developed a set of requirements eliciting tools to reflect goal models into UML diagrams [6], where Class, Sequence and Collaboration diagrams are considered. According to the horse shoe model, this corresponds to the forward engineering phase. In this paper, we consider statecharts [14] as a suitable intermediate representation for the dynamic behavior of legacy code, as well as the abstract interface to the environment.

This paper is not the first attempt to discover goal models from sources other than requirements. In the KAOS project, goal models can be inferred from user scenarios [33]. However, scenarios generally do not cover all possible paths of program executions. And legacy software often comes with incomplete and inaccurate documents. Therefore our methodology complements the KAOS approach, based on the idea of understanding-by-refactoring. The recovered goal model is not guaranteed to capture the intentions of the original requirements, but can be trusted to capture the implemented intentions of stakeholders, as understood by programmers. It is also more traceable from the code since each refactoring step is documented and is also invertible.

Goal models can also be seen as abstractions of software processes. Other literature details techniques for recovering process models from events collected during the software development process [4]. However, this work focuses on inferring the processes used to develop software, rather than the processes realized by the software itself. Program model checking [34] systems, such as Bandera [5], extract finitestate machines from Java source code. Although such systems have succeeded in finding counter examples for some programs, the combinatorial explosion of states ultimately limits their applicability in revealing intentions behind a large software system. Not surprisingly we found a similar combinational barrier when requirements goal models are converted into state machines for model checking [13]. According to our case study, goal models can be built more concisely from statecharts.

In [29] and later in [30], an algorithm is proposed to compress state diagrams into UML state diagrams, which constitute a variant of the statechart notation. The algorithm works by using execution event traces. The approach is complementary to our technique, which does not rely on program inputs.

Pattern-based design recovery as proposed in [23] finds UML diagrams, including collaboration diagrams and statecharts from source code. This approach is also similar to our work, but relies on pattern matching rather than legacy code comments.

\section{Conclusions and future work}

We have proposed a framework for reverse engineering legacy code in order to discover the requirements goals it was intended to fulfill. Our tool has been illustrated with two case studies involving public-domain legacy email systems (SquirrelMail and Columba). The case studies suggest that the process of recovering requirements goals can be systematized. Moreover, the reverse engineered goal models are traceable in the code, making it feasible to forward engineering goal models into new architectures.

In future work, we propose to study methods for the recovery of softgoals using hints from architecture and design documents. We will also compare the reverse engineered goal models with the goal models derived through requirements elicitation.

\section{References}

[1] R. Castello. SquirrelMail 1.5.0, http://www.squirrelmail.org.

[2] E. J. Chikofsky and J. H. C. II. Reverse engineering and design recovery: A taxonomy. IEEE Software, 7(1):13-17, 1990.

[3] L. Chung, B. A. Nixon, E. Yu, and J. Mylopoulos. NonFunctional Requirements in Software Engineering. Kluwer Academic Publishing, 2000. 
[4] J. Cook and A. Wolf. Discovering models of software processes from event-based data. ACM Transactions on Software Engineering and Methodology, 7(3):215-249, 1998.

[5] J. Corbett, M. Dwyer, J. Hatcliff, C. Pasareanu, Robby, S. Laubach, and H. Zhang. Bandera: Extracting finite-state models from java source code. In ICSE00, pages 439-448. ACM Press.

[6] L. M. Cysneiros and J. C. S. P. Leite. Non-functional requirements: from elicitation to conceptual models. IEEE Transactions on Software Engineering, 30(5):328-350, May 2004.

[7] A. Dardenne, A. van Lamsweerde, and S. Fickas. Goaldirected requirements acquisition. Science of Computer Programming, 20(1-2):3-50, Apr. 1993.

[8] E. H. D'Hollander, F. Zhang, and Q. Wang. The Fortran parallel transformer and its programming environment. Journal of Information Sciences, (106):293-317, 1998.

[9] F. Dietz and T. Stich. The Columba project, 1.0 RC2, http://columba.sourceforge.net.

[10] J. Ferrante, K. Ottenstein, and J. Warren. The program dependence graph and its use in optimization. ACM Trans. Program Languages and Systems, 9(3):319-349, 1987.

[11] S. Fickas, L. Ehlhardt, M. Sohlberg, and B. Todis. Towards personal RE: A challenging case study, 45-02. Technical report, Computer Science Department, University of Oregon.

[12] M. Fowler. Refactoring: Improve the design of existing code. Addison-Wesley, Reading MA, 1997.

[13] A. Fuxman, L. Liu, J. Mylopoulos, M. Pistore, M. Roveri, and P. Traverso. Specifying and analyzing early requirements in Tropos. In $R E^{\prime} 03$, pages 105-114, 2003.

[14] D. Harel. Statecharts: A visual formalism for complex systems. Science of Computer Programming, pages 231-274, 1987.

[15] D. Harel and A. Naamad. The STATEMATE semantics of statecharts. ACM Trans. on Software Engineering and Methodology, 5(4):293-333, Oct. 1996.

[16] S. Horwitz, T. Reps, and D. Binkley. Interprocedural slicing using dependence graphs. ACM Trans. On Programming Languages and Systems, 12(1):26-60, 1990.

[17] B. Hui, S. Liaskos, and J. Mylopoulos. Goal skills and preference framework. In $R E^{\prime} 03$, pages 117-126.

[18] R. Kazman, S. G. Woods, and S. J. Carriere. Requirements for integrating software architecture and reengineering models: CORUM II. pages 154-163, 1998.

[19] J. Leite. Working results on software re-engineering. ACM SIGSOFT Software Engineering Notes, 21(2):39-44, 1996.

[20] T. Mens and T. Tourwe. A survey of software refactoring. IEEE Trans. Software Engineering, 30(2):126-139, 2004.

[21] H. Muller, J. Jahnke, D. Smith, M. Storey, S. Tilley, and K. Wong. Reverse engineering: A roadmap. In Future of Software Engineering, ICSE'O0, pages 49-60, 2000.

[22] J. Mylopoulos, L. Chung, and B. Nixon. Representing and using nonfunctional requirements: A process-oriented approach. IEEE Transactions on Software Engineering, 18(6):483-497, Jun 1992.

[23] J. Niere, J. P. Wadsack, and A. Zundorf. Recovering UML diagrams from Java code using patterns. In SCASE'01, 2001.

[24] W. Opdyke. Refactoring: A program restructuring aid in designing object-oriented application frameworks. $\mathrm{PhD}$ thesis, 1992.
[25] K. Otteinstein and L. Ottenstain. The program dependence graph in a software development environment. ACM SIGPLAN Notices, 19(5):177-184, May 1984.

[26] S. Owre, J. Rushby, N. Shankar, , and F. von Henke. Formal verification for fault-tolerant architectures: Prolegomena to the design of PVS. IEEE Trans. Software Eng., 21(2):107125, Feb. 1995.

[27] M. Samek. Practical statecharts in $C / C++$. Quantum programming for embedded systems. CMP books, 2002.

[28] N. Shankar. Steps towards mechanizing program transformations using PVS. Science of Computer Programming, 26(1-3):33-57, May 1996.

[29] T. Systa. Understanding the behavior of Java programs. In WCRE'O0, pages 214-223, 2000.

[30] T. Systa, K. Koskimies, and E. Makinen. Automated compression of state machines using uml statechart diagram notation. Information \& Software Technology, 44(10):565578, 2002.

[31] F. Tip. A survey of program slicing techniques. Journal of programming languages, 3:121-189, 1995.

[32] A. van Lamsweerde. Goal-oriented requirements engineering: From system objectives to UML models to precise software specifications. In ICSE 2003, pages 744-745, 2003.

[33] A. van Lamsweerde and L. Willemet. Inferring declarative requirements from operational scenarios. IEEE Trans. Software Engineering, 24(12):1089-1114, Nov. 1998.

[34] W. Visser, K. Havelund, G. Brat, S. Park, and F. Lerda. Model checking programs. ASE, 2002.

[35] M. Weiser. Program slicing. IEEE Trans. Software Engineering, 10(4):352-357, July 1984.

[36] www.eclipse.org. Eclipse IDE 3.0.1: Refactoring in JDT, EMF, UML2.

[37] F. Zhang and E. H. D'Hollander. Using hammock graphs to structure programs. IEEE Trans. Software Engineering, 30(4):231-245, Apr. 2004. 